\title{
La datation des eaux souterraines par analyse des CFC : un outil de gestion durable de la ressource en eau
}

\author{
V. VERGNAUD-AYRAUD', L. AQUILINA², H. PAUWELS ${ }^{3}$, Th. LABASQUE²
}

Mots-clés : âge de l'eau, nitrate, aquifères de socle, laboratoire, CFC, gestion

\section{Introduction}

Au sein des aquifères, l'âge des eaux souterraines, c'est-à-dire la durée de son temps de transfert de son point d'infiltration vers son exutoire naturel ou non (captage) peut varier du jour au(x) million(s) d'années suivant le contexte géologique et hydrogéologique local [KIPFER, 2001]. L'estimation de l'âge de l'eau est donc une information de premier ordre pour connaître le temps de renouvellement de l'eau dans la nappe, calibrer les modèles hydrogéologiques, prédire la vulnérabilité de la nappe aux pollutions, et de manière globale pour assurer une gestion efficace de l'aquifère.

La détermination du temps de résidence des eaux souterraines est souvent réalisée de manière indirecte à partir de la résolution des équations de Darcy ou de la Loi de Fick [METCALFE et al. 1998]. Mais cette méthode nécessite une connaissance exhaustive du milieu souterrain et de ses propriétés hydrauliques pour limiter l'incertitude sur les âges. Lapproche directe à l'aide d'un traceur permet une observation plus objective des temps de résidence au sein de l'aquifère, à l'échelle du bassin-versant.

Il existe différents types de traceurs adaptés aux différents ordres de grandeurs qui peuvent être rencontrés dans le milieu souterrain. Le plus connu est sans conteste le carbone14 $\left({ }^{14} \mathrm{C}\right)$ utilisé pour des temps de résidences compris entre quelques centaines d'années et 50000 ans. D’une manière générale, ces

1 LADES, Laboratoire de datation des eaux souterraines, Rennes (35). Mél. : contactalades.fr

2 UMR Géosciences, Université de Rennes 1.

3 Département Eau, BRGM, Orléans. nappes à très faible taux de renouvellement (parfois abusivement qualifiées de nappes fossiles) sont plutôt aujourd'hui bien identifiées car elles sont souvent des aquifères relativement productifs et très utilisés. Un second traceur largement utilisé en hydrogéologie est le tritium dont la concentration atmosphérique a été marquée par un pic durant le début des années 60 , à la suite des essais nucléaires atmosphériques. Les concentrations sont aujourd'hui beaucoup plus basses et les mesures sont devenues beaucoup plus délicates à interpréter. Les chlorofluorocarbones (CFC) sont eux caractéristiques des eaux récentes, infiltrées au cours des 50 dernières années. Ce sont donc des traceurs contemporains de l'intensification des activités anthropiques, tant au niveau agricole que technologique.

La méthode de datation des eaux souterraines à partir de l'analyse des CFC a été appliquée au contexte des aquifères de socle (nappes des roches cristallines telles que le granite ou le schiste) au cours d'une thèse à l'université de Rennes 1 (AYRAUD, 2006 ; financement Brgm, CNRS et région Bretagne). Cet article présente donc, après un bref rappel du principe de datation à l'aide des $\mathrm{CFC}$, les résultats obtenus au cours de cette thèse. La dernière partie mettra en avant l'intérêt de la connaissance de l'âge de l'eau dans les études hydrogéologiques réalisées dans le cadre de la gestion des ressources en eau.

\section{La datation par analyse des CFC}

\subsection{Les traceurs utilisés : les CFC}

Les CFC ou chlorofluorocarbones sont plus connus sous leur nom commercial : fréons ${ }^{\odot}$. La production de ces gaz a commencé au cours des années 50 dans 
un premier temps pour remplacer les gaz réfrigérants habituels plutôt dangereux et toxiques (ammoniaque, isobutane...). Ils ont eu depuis de nombreuses autres applications comme gaz propulseur des aérosols, solvants, matière première dans la synthèse de composés synthétiques, agent d'expansion de mousse... [HÖHENER et al., 2003].

Il n'existe non pas un mais des CFC suivant les combinaisons des atomes de chlore et de fluor dont ils sont composés. Chaque CFC est donc repéré par un chiffre de type xyz où :

- $\mathrm{x}$ est le nombre d'atome de carbone -1 (pour 1 atome de carbone x étant égal à zéro n'est pas noté et le chiffre devient yz uniquement),

- y est le nombre d'atome d'hydrogène +1 ,

- z est le nombre d'atome de fluor.

le nombre d'atomes de chlore est déduit de la séquence, chaque atome de carbone possédant quatre liaisons possibles.

Le CFC-11 est donc une molécule avec un atome de carbone, pas d'atome d'hydrogène, un atome de fluor et trois atomes de chlore. Le nombre de combinaisons est très important mais cinq CFC sont plus largement utilisés. La figure 1 présente les formules chimiques des trois CFC utilisés en datation des eaux.

Les CFC de par leur innocuité pour la population humaine (ininflammables, non toxiques, ni cancérigènes) ont été très largement utilisés. En conséquence, les concentrations atmosphériques de ces gaz ont très fortement augmenté dans l'atmosphère à partir des années 1960. Néanmoins dès 1974, des études scientifiques ont mis en évidence que le radical $\mathrm{Cl}$ issu de la photolyse de ces composés pouvait avoir un effet destructeur sur la couche d'ozone, rempart naturel de la Terre contre les UV solaires. À partir de
1978, la NOAA (National Oceanic and Atmospheric Administration) a lancé un programme de suivi des composés halogénés (dont font parti les CFC) dans l'atmos-phère. Dès 1985, la production de ces composés a été réglementée au travers du protocole de Montréal. La fin des CFC est annoncée dans les années 1990 lorsqu'ils sont classés dans la catégorie des gaz à effet de serre. Ils sont également soumis au protocole de Kyoto ratifié en 1997.

Le fort engouement industriel pour ces gaz « miracles » puis la découverte de leur(s) propriété(s) néfaste(s) pour l'environnement ont conduit à une évolution caractéristique des concentrations atmosphériques de ces composés (figure 2) : une croissance exponentielle entre 1950 et 1990, avant un plateau des années 1990 aux années 2000 suivi d'une légère diminution à partir des années 2000.

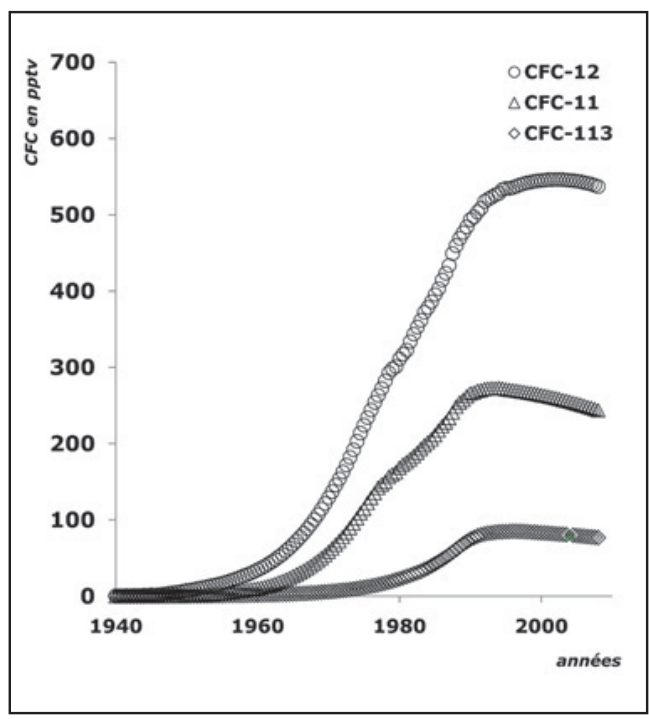

Figure 2. Évolution des concentrations atmosphériques des CFC-12, CFC-11 et CFC-113 entre 1940 et 2005. Les concentrations sont exprimées en pptv (partie par trillions de volume) soit $0,0001 \mu \mathrm{l} / \mathrm{l}$. Données issues de [WALKER et al., 2000]

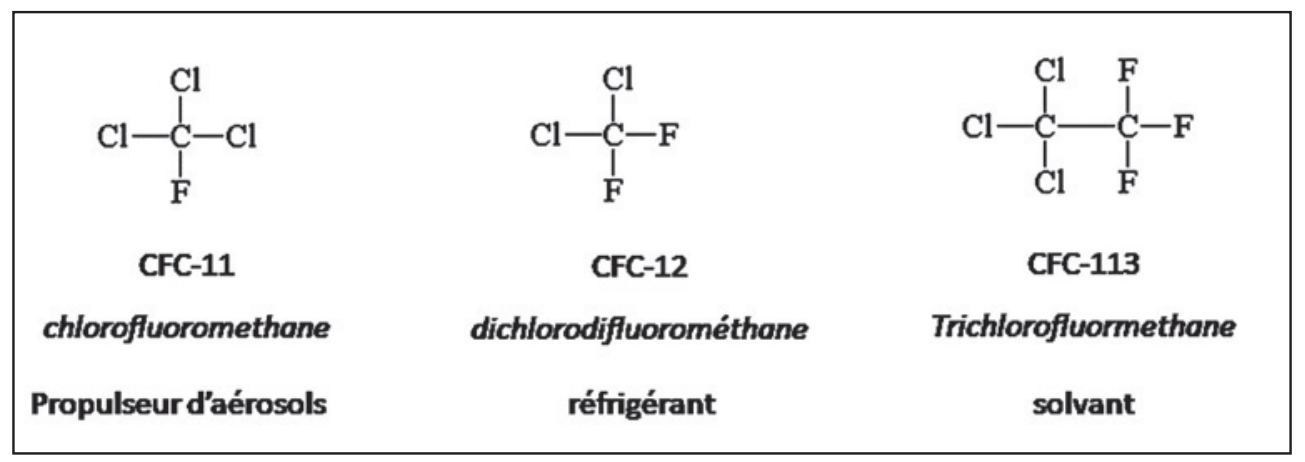

Figure 1. Formule, dénomination et utilisation principale des trois CFC utilisés pour la datation des eaux souterraines 


\subsection{Le principe de la datation}

En théorie, le principe de datation par traceurs atmosphériques tels que les CFC est assez simple : l'eau qui s'infiltre dans le sous-sol se met à l'équilibre avec l'atmosphère, elle a donc une signature chimique en lien direct avec la composition de l'atmosphère.

Dans le milieu souterrain, on admet communément (en tout cas dans les zones de recharge) et par simplification que la circulation des eaux souterraines est de type « circulation par effet piston » : dans ce type de circulation, il n'y a que peu de mélange entre les masses d'eau infiltrées au cours de pluies différentes, et $a$ fortiori au cours d'années différentes. De manière très imagée, on peut assimiler la circulation des eaux souterraines à un collier de perles ou chaque nouvelle pluie infiltrée (perle) vient pousser celle précédemment en place, aboutissant, en bout de chaîne, à la libération d'une masse d'eau au niveau de l'exutoire. Tout l'intérêt de la datation des eaux réside dans le fait de savoir combien de « perles » contient le « collier » représentant la circulation des eaux souterraines. Pour des situations plus complexes, un échantillon d'eau peut représenter les contributions composites de plusieurs zones de contribution ayant chacune son temps de résidence propre. Dans ce cas, la distribution des temps de résidence au sein de l'aquifère est une mesure tout à fait importante, même si elle ne représente plus directement le temps de résidence mais un âge apparent.

Pour suivre une masse d'eau il suffit de la « marquer » à l'aide de traceurs (figure 3). La masse d'eau qui s'infiltre va garder tout au long de son trajet souterrain sa « signature atmosphérique » jusqu'à son exutoire naturel (source, rivière) ou artificiel (captage, forage). Plusieurs gaz atmosphériques composent cette « signature atmosphérique » de l'eau, mais il convient d'utiliser comme traceur un (ou des) composés qui sont : - peu sensibles à la dégradation et à l'adsorption dans le milieu naturel,

- et dont les concentrations d'une année sur l'autre sont connues et varient suffisamment pour être distinguées.

Les chlorofluorocarbones ont été identifiés depuis les années 1970 en océanographie et le début des années 1980 en hydrogéologie, comme répondant à ces contraintes et représentent donc des traceurs adéquats des masses d'eau.

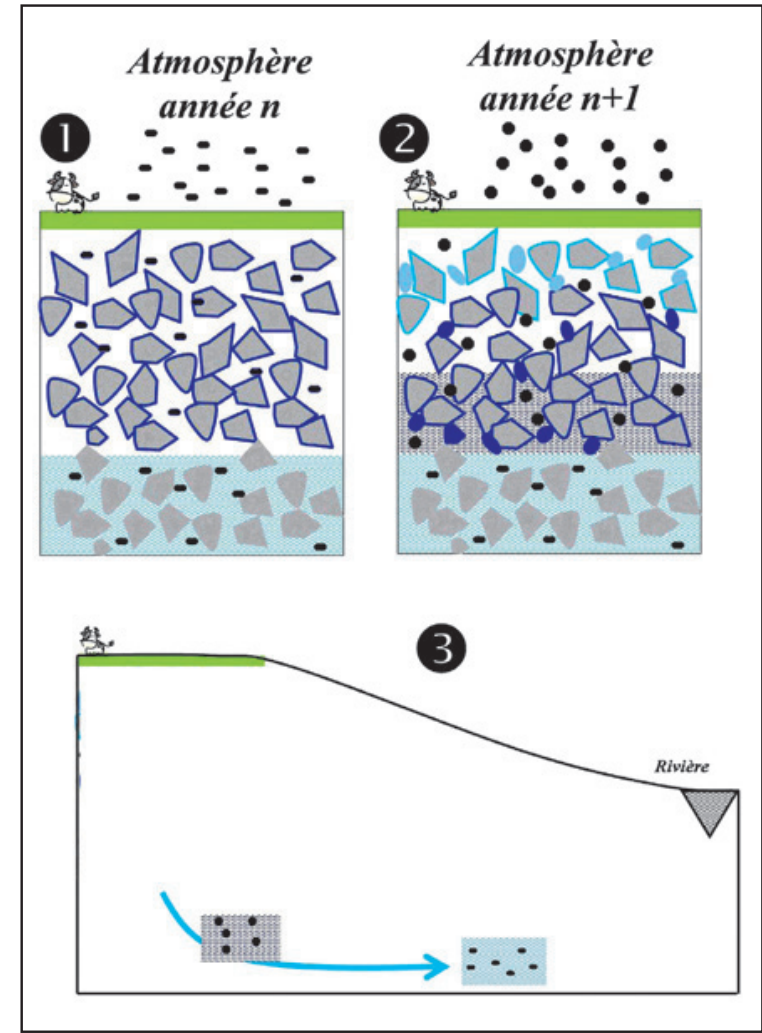

Figure 3. Représentation simplifiée de la circulation d'eaux souterraines marquées par transfert « piston ».

1) La première année, l'eau des précipitations s'infiltre dans le milieu et acquiert la signature chimique de l'atmosphère (symboles identiques).

2) L'année suivante, la concentration atmosphérique du traceur augmente, l'eau qui s'infiltre au cours de cette année a donc une signature chimique différente de celle de l'année précédente.

3) Ces signatures chimiques vont, en l'absence de dilution, de mélange ou de dégradation, être conservées par la masse d'eau jusqu'à l'exutoire du système.

Le succès de la datation des eaux souterraines à partir du dosage des CFC repose sur quatre conditions principales.

(1) Laquifère n'est pas contaminé en CFC par des sources anthropiques (décharges sauvages où auraient pu être stockés des réfrigérateurs, sites situés à proximité d'une zone industrielle utilisant ou ayant utilisé des CFC dans leurs processus industriels...); néanmoins, il est assez rare que le site soit contaminé pour tous les CFC analysés, chacun ayant un usage spécifique (réfrigérant, solvant ou gaz propulseur).

(2) Les concentrations en CFC n'ont pas été altérées par des processus biologiques, géochimiques ou hydrologiques ayant conduit par dilution ou dégradation à une baisse significative des concentrations en CFC dans l'eau. À noter que les CFC sont des composés peu dégradables et que les processus de dégradation 
biologique sont séquentiels. La dégradation de tous les CFC analysés nécessite des conditions de milieu extrêmement réductrices qui sont rarement rencontrées dans les milieux souterrains. L'utilisation de plusieurs CFC permet d'éviter que la dégradation d'un CFC compromette toute datation. Les dilutions sont souvent déjà quantifiées par la ou les étude(s) hydrogéologique(s) réalisées sur le site, ce qui permet de s'en affranchir.

3 La température moyenne du sol doit pouvoir être estimée pour calculer le facteur de solubilité.

4 La méthode de prélèvement doit permettre un échantillonnage sans contact atmosphérique ou avec toute autre source de CFC.

\subsection{L'analyse des CFC}

Les concentrations atmosphériques en CFC sont faibles : de l'ordre du $\mu$ pour $1000 \mathrm{l}$ d'air. D'après les lois de solubilité des gaz entre l'eau et l'air, les concentrations en CFC dans les eaux souterraines sont 100 à 1000 fois moins importantes : de l'ordre de la picomole par litre $\left(10^{-12} \mathrm{~mol} / \mathrm{l}\right)$. Lanalyse des CFC nécessite donc des outils analytiques de pointe.

Lanalyse des CFC dans les eaux souterraines est réalisée en trois étapes (figure 4):

- l'extraction des gaz dissous dans l'eau souterraine par dégazage (bullage à l'azote pur),

- la préconcentration des gaz sur un piège (piégeage à froid),

- la desorption à chaud des gaz pour analyse en chromatographie en phase gazeuse.

Le système de préconcentration, appelé « purge-andtrap » permet de concentrer en un faible volume (quelques millilitres) l'ensemble des gaz de l'échantillon (30 ml d'eau) et d'atteindre ainsi une limite de détection extrêmement faible.

\section{Application des résultats au transfert d'azote dans les aquifères fracturés hétérogènes (exemple de la Bretagne)}

Les concentrations en nitrates dans les aquifères sont très variables même pour un contexte agricole identique et un prélèvement à une profondeur similaire (figure 5). Lacquisition de données sur le temps de résidence des eaux souterraines est indispensable pour mieux comprendre les flux d'azote dans le milieu. En effet, l'absence de nitrate est-elle liée à un temps de résidence plus important ou à des processus naturels d'autoépuration ? La gestion de ces aquifères découle en grande partie de la réponse à cette question.

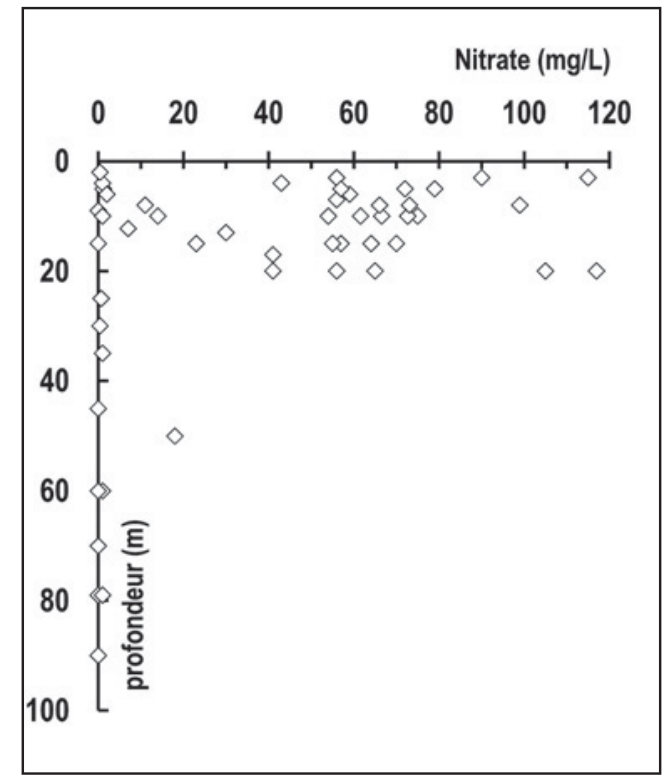

Figure 5. Évolution des concentrations en nitrates en fonction de la profondeur de prélèvement sur différents sites en Bretagne [AYRAUD, 2006]

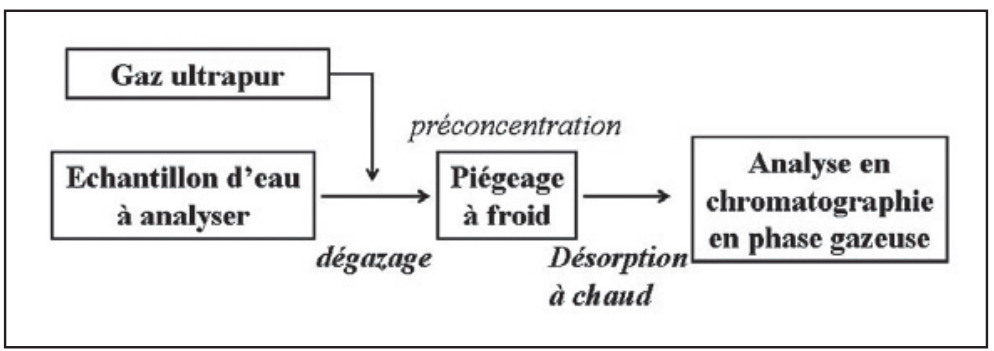

Figure 4. Étapes de l'analyse des CFC 


\subsection{Les spécificités géologiques et hydrogéolo- giques des aquifères de socle}

Les roches cristallines de type granite ou schiste (entre autres) ont une porosité intrinsèque assez faible. Dans ces milieux la circulation de l'eau s'effectue principalement au gré des fissures, fractures et failles qui recoupent ces roches de manière hétérogène (d'où le terme d'aquifères fracturés hétérogènes). Lors de leur altération, ces roches forment un matériau argilo-sableux qui acquiert des caractéristiques proches des milieux poreux. Les aquifères de socles peuvent donc être découpés, de manière simplifiée, en deux zones géologiques et hydrogéologiques distinctes séparées par une zone intermédiaire de transition (figure 6).

En amont des bassins-versants, la zone altérée argilosableuse peut atteindre une épaisseur de quelques dizaines de mètres. La structure poreuse du matériel géologique qui la compose lui confère des propriétés capacitives importantes. Il est courant qu'une nappe de surface se développe dans cette couche géologique, sa productivité étant dépendante de la proportion d'argile qu'elle contient. Proches de la surface, ces nappes sont très sensibles aux conditions climatiques et le battement de nappe (amplitude entre le niveau le plus bas et le plus haut) peut atteindre la dizaine de mètres. Elles sont de plus fortement influencées par les activités de surface.

Sous cette zone altérée, la roche saine est entaillée de fissures et fractures dont la densité diminue avec la profondeur. Suivant la connectivité entre ces fractures un aquifère plus ou moins productif peut exister. Néanmoins, cet aquifère et les circulations d'eau qui y sont associées sont extrêmement localisés.

Entre ces deux zones aux caractéristiques hydrogéologiques contrastées, il existe une zone de transition estimée à quelques mètres d'épaisseur où la densité de fracture est si intense que le milieu fracturé se comporte comme un milieu poreux.

Lépaisseur de chacune de ces zones et plus particulièrement de la zone altérée, varie d'un site à un autre avec même pour certains cas, une absence de zone altérée.

\subsection{Les temps de résidence des eaux souterraines}

Au cours de cette étude, une cinquantaine de points répartis sur sept sites ont été échantillonnés et analysés. La figure 7 présente les âges obtenus à partir de l'analyse des CFC en fonction de leur profondeur. La différence de comportement hydrogéologique entre les deux zones identifiées dans les aquifères de socle apparait nettement dans l'évolution des âges en fonction de la profondeur. Dans la zone altérée, l'âge des eaux souterraines s'étend de quelques années dans les zones de recharge (zones d'infiltration préférentielle en amont) à 25 ans environ. Le fait que dans cette partie altérée aucun âge plus important n'ait été observé est probablement lié à l'extension relativement faible des nappes de surface des aquifères de socles qui ne permet pas de boucles de

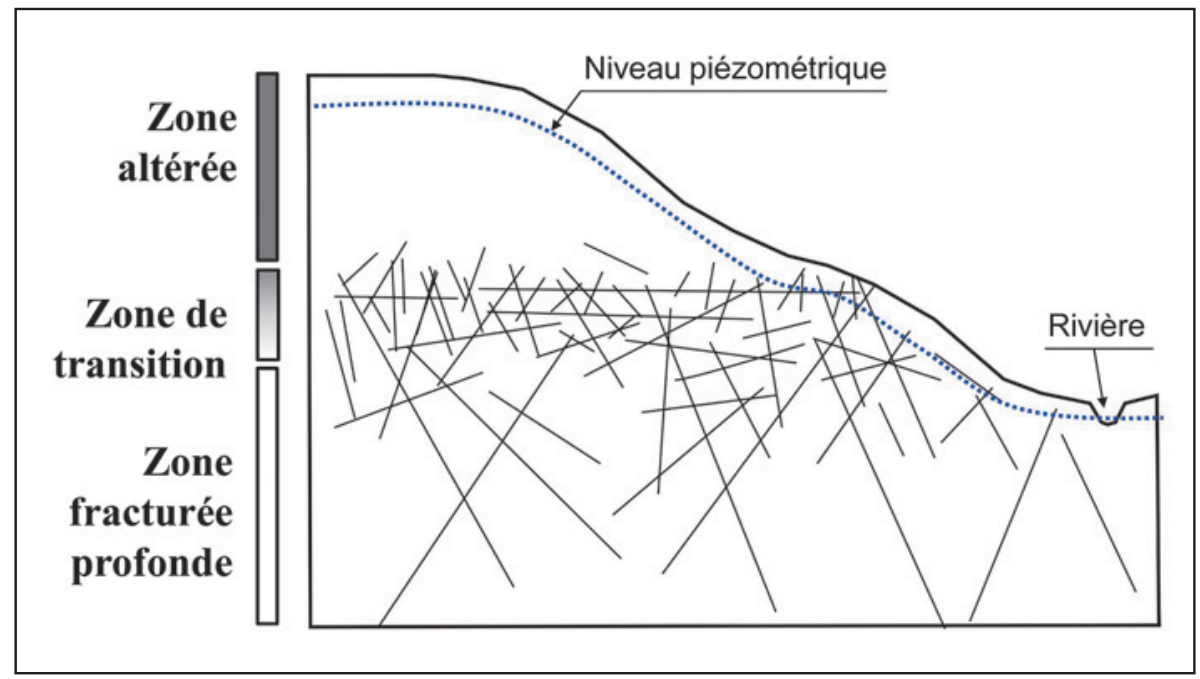

Figure 6. Schéma simplifié des aquifères de socles. Inspiré de [WYNS et al., 2004] 
circulation d'une longueur supérieure à 25 ans. De plus, l'absence d'eau avec un temps de résidence compris entre 5 et 15 ans dans la zone altérée peut être expliquée par la difficulté de dater précisément les eaux très récentes à l'aide des CFC. En effet entre 1990 et 2005, les concentrations atmosphérique en CFC ont cessé d'augmenter et certaines ont même amorcé une diminution. Il est devient difficile sur cette période très récente de discriminer une année d'une autre. Sur cette zone, des analyses sont en cours à l'aide d'un autre traceur : le SF6 (hexafluorure de soufre). Ce gaz, également d'origine anthropique, est apparu dans les années 1970 (c'est un isolant électrique) et présente une augmentation continue de ses concentrations atmosphériques depuis cette date. Il permet donc la datation des eaux souterraines très récentes (15 dernières années) avec plus de précision que les CFC.

Dans la zone fissurée, les âges des eaux souterraines qui ne sont pas situées dans une zone d'appel de pompage présentent toutes des temps de résidence supérieurs à 15-20 ans. Ceci indique que la zone altérée est la zone de recharge principale de l'aquifère fissuré sous-jacent.

Concernant les vitesses de transfert, dans la zone altérée, l'hétérogénéité des sites échantillonnés ne permet pas de distinguer une tendance générale. À l'inverse dans la zone fissurée, une vitesse apparente de transfert moyenne de l'ordre de $3 \mathrm{~m}$ par an est



Figure 7. Relation profondeur / âge des eaux souterraines observée sur les sites hors zone de pompage. Ceci conduit à penser que, tandis que les nappes de surface dans la zone altérée sont directement dépendantes de la topographie du site et des pentes locales, les nappes de la zone fissurées semblent, elles, influencées par un facteur plus régional identique sur chaque site.

Cette étude met aussi en évidence les conséquences en termes de temps de résidence d'un pompage dans la nappe souterraine. En effet une zone exploitée pour l'alimentation en eau potable a été échantillonnée et il apparaît clairement un décalage des âges entre les eaux de la zone fissurée de ces puits influencés par un pompage et les autres puits échantillonnés à même profondeur. Ce rajeunissement des eaux en profondeur est une conséquence de la mise en exploitation du site. Les conséquences vont être différentes suivant l'historique des apports anthropiques sur le site et ses capacités auto-épuratrices. Néanmoins, cette analyse met en avant un possible déséquilibre lié à l'exploitation ; pour utiliser la ressource de façon durable, il serait donc nécessaire de faire des analyses régulières de façon à s'assurer qu'un nouvel équilibre a été atteint et que ce rajeunissement n'est pas la signe d'une sur-exploitation de la nappe.

\subsection{Apports de la datation sur la compréhension du transfert d'azote}

La question principale de cette étude était de mieux comprendre les transferts d'azote dans les bassinsversants et plus particulièrement d'avoir un outil permettant de mettre en évidence la vulnérabilité des nappes.

La figure 8 met clairement en évidence la relation entre l'âge des eaux souterraines et leur contamination potentielle en nitrates ; ainsi l'intensification des pratiques agricoles amorcée au cours des années 1970-1980 est clairement accompagnée par une augmentation forte des concentrations en nitrates chargés il y a une vingtaine d'années. Néanmoins, certaines des eaux infiltrées à la même période ont de faibles concentrations en nitrates malgré un environnement agricole. Ces résultats indiquent une bonne capacité d'autoépuration naturelle de ces nappes, ce qui laisse présager une bonne protection de l'aquifère.

À l'inverse, il n'est pas observé de réelle tendance à la diminution des concentrations en nitrates dans les 


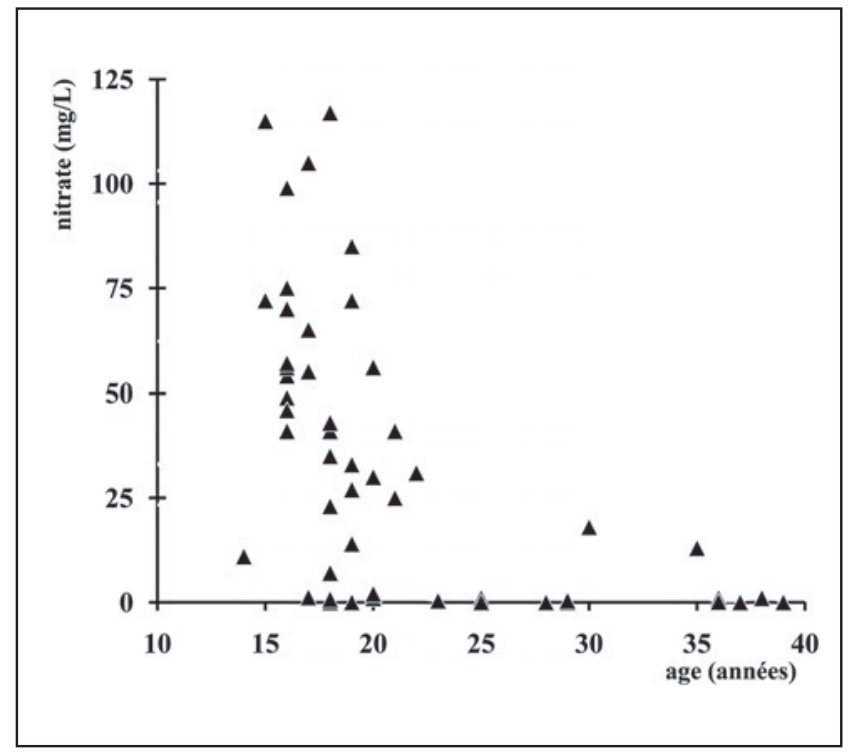

Figure 8. Relation concentrations en nitrates / âge des eaux souterraines

eaux plus récentes mais plutôt une stabilisation. Néanmoins le nombre de données disponibles sur les eaux récentes est trop limité pour en tirer de réelles conclusions et il est nécessaire pour cette problématique de travailler à l'échelle du site car les mesures de protection de l'environnement sont très différentes d'un bassin versant à l'autre.

\section{L'âge des eaux souterraines : un outil de gestion de la ressource en eau pour les collectivités et les bureaux d'études}

Létude réalisée au cours de ce projet universitaire met en évidence l'intérêt de la connaissance de l'âge de l'eau dans la gestion durable de la ressource. En effet, la datation des eaux souterraines permet d'apporter des éléments de réponse aux questions essentielles de la gestion de l'eau :

- Quel est le taux de renouvellement de la nappe?

- De quand datent les polluants présents dans la nappe (et en particulier de quand datent les apports de nitrates à la nappe) ?

- Quel délai va-t-il exister entre un changement de pratiques en surface et un changement de la qualité de l'eau?

Au-delà, c'est à la recommandation du bon état écologique des eaux en 2015 requise par la directive cadre européenne, que la datation des eaux souterraines peut permettre de contribuer.

Lanalyse des concentrations en CFC a également un second avantage non négligeable dans la gestion des bassins-versants : c'est un excellent traceur des eaux récentes car spécifique des eaux infiltrées après les années 1950. Leur présence dans une nappe « ancienne », ou leur apparition progressive au cours d'un essai de pompage longue durée sont les signes de connexions de l'aquifère exploité avec les eaux de surface et donc d'une vulnérabilité potentielle de la ressource.

\section{Conclusion}

Longtemps réservée au domaine de la recherche universitaire, la datation des eaux souterraines par le dosage des CFC est désormais accessible pour tous les sites depuis la création en France d'un Laboratoire de datation des eaux souterraines ${ }^{4}$ (LADES), issu des travaux présentés dans cet article. Contrairement aux autres méthodes de datation telles que le tritium/hélium, la datation par analyse des CFC a pour avantage d'être relativement peu onéreuse et de nécessiter des délais d'analyses courts (10 à 15 jours entre le prélèvement et le résultat). De plus, le laboratoire bénéficie des dernières avancées scientifiques sur la datation avec l'utilisation du traceur pour les eaux très récentes : le SF6.

De plus, on peut constater dans la création de ce laboratoire un bel exemple de valorisation des travaux de recherche et d'un travail de thèse pour les besoins des industriels et des collectivités territoriales.

\section{Remerciements}

La thèse de Virginie VERGNAUD a été financée par le BRGM. Le projet de datation des eaux a été soutenu financièrement et matériellement par le CNRS, l'université de Rennes 1 et la région Bretagne.

Les auteurs tiennent à remercier l'ensemble des acteurs ayant participé de près ou de loin à ce projet et plus particulièrement, les personnels en charge des sites sur lesquels ont été effectués les échantillonnages pour leur disponibilité.

\footnotetext{
4 LADES - Espace Performance Bâtiment C1-C2 35769 SaintGrégoire. www.lades.fr
} 


\section{Bibliographie}

HÖHENER P., WERNER D. et al. (2003). “Worldwide occurence and fate of chlorofluorocarbons in groundwater." Critical Reviews in Environmental Science and Technology 33(1): 1-29.

KIPFER R. (2001). "Les voyages intertemporels des eaux souterraines". EAWAG News, $49: 12-14$

METCALFE R., HOOKER P.J. et al. (1998). "Dating quaternary groundwater flow events : a review of available methods and their application". Dating and duration of fluid flow and fluid rock interaction. J. Parnell. London, Geological Society. 144: 233-260.
WALKER S. J., WEISS R.F. et al. (2000). "Reconstructed histories of the annual mean atmospheric mole fraction for the halocarbons CFC-11, CFC-12, CFC113 , and carbon tetrachloride." Journal of Geophysical Research 105(C6): 14285-14296.

WYNS R., BALTASSAT J.-M., et al. (2004). "Application of magnetic resonance soundings to groundwater reserve mapping in weathered basement rocks (Brittany, France)." Bull. Soc. géol. Fr 175(1): 21-34.

\section{Résumé}

\section{VERGNAUD-AYRAUD, L. AQUILINA,}

H. PAUWELS, Th. LABASQUE. La datation des eaux souterraines par analyse des CFC : un outil de gestion durable de la ressource en eau L'âge des eaux souterraines est un facteur clé dans la gestion de la ressource en eau : il est un excellent indicateur de la vulnérabilité des nappes aux pollutions. Depuis les années 1980, les chlorofluorocarbones ICFC ou fréons, gaz anthropiques utilisés entre autres comme réfrigérant] sont utilisés dans la recherche en hydrogéologie comme traceurs des eaux souterraines récentes (rechargées au cours des 50 dernières annéesl. Leur utilisation dans le cadre d'une étude sur le fonctionnement des aquifères de socle a mis en évidence leur utilité dans la compréhension du fonctionnement hydrogéologique des aquifères (influence directe du pompage sur le taux de renouvellement de la nappe) et le transfert de la pollution azotée liée aux nitrates. « De quand datent les nitrates présents dans la nappe ? « Quel est le taux de renouvellement de l'aquifère » : autant de questions indispensables pour une gestion durable de la ressource en eau, auxquelles la datation des eaux souterraines par les CFC apporte des éléments de réponses.

\section{Summary}

V. VERGNAUD-AYRAUD, L. AQUILINA,

H. PAUWELS, Th. LABASQUE. Groundwater age determination by CFC analysis: a tool for groundwater sustainable management Groundwater age is a key parameter in groundwater management: it is a clear indicator of aquifer pollution vulnerability. Hydrogeological academic research from the ' $80 \mathrm{~s}$ uses chlorofluorocarbons (CFC or Freons, anthropic gases used as refrigerants) as tracers for recent groundwater linfiltrated in the last 50 years). The results of a study on hard-rock aquifer functioning show that they are very useful for aquifer hydrogeological functioning lpumping influence on groundwater renewal ratel and for the understanding of nitrate pollution behavior. "How old are the nitrates in ground water?" «What is the aquifer renewal rate? »: these are questions that groundwater age determination by CFC analysis could help answer, improving sustainable groundwater management. 